\title{
Molecules in the circumnuclear disk of the Galactic center
}

\author{
Nanase Harada ${ }^{1}$, Denise Riquelme ${ }^{1}$, Serena Viti ${ }^{2}$, Karl Menten ${ }^{1}$, \\ Miguel Requena-Torres ${ }^{1}$, Rolf Güsten ${ }^{1}$ and Stefan Hochgürtel ${ }^{1}$ \\ ${ }^{1}$ Max Planck Institute for Radio Astronomy, Auf dem Hügel 69, D-53121, Bonn, Germany \\ email: harada@mpifr-bonn.mpg.de \\ ${ }^{2}$ University College London, Department of Physics and Astronomy, Gower Street, London
} WC1E 6BT, UK

\begin{abstract}
Within a few parsecs around the central black hole Sgr A*, chemistry in the dense molecular cloud material of the circumnuclear disk (CND) can be affected by many energetic phenomena such as high UV-flux from the massive central star cluster, X-rays from Sgr A*, shock waves, and an enhanced cosmic-ray flux. Recently, spectroscopic surveys with the IRAM 30 meter and the APEX 12 meter telescopes of substantial parts of the $80-500 \mathrm{GHz}$ frequency range were made toward selected positions in and near the CND. These data sets contain lines from the molecules $\mathrm{HCN}, \mathrm{HCO}^{+}$, $\mathrm{HNC}$, CS, SO, $\mathrm{SiO}, \mathrm{CN}, \mathrm{H}_{2} \mathrm{CO}, \mathrm{HC}_{3} \mathrm{~N}, \mathrm{~N}_{2} \mathrm{H}^{+}, \mathrm{H}_{3} \mathrm{O}^{+}$ and others. We conduct Large Velocity Gradient analyses to obtain column densities and total hydrogen densities, $n$, for each species in molecular clouds located in the southwest lobe of the CND. The data for the above mentioned molecules indicate $10^{5} \mathrm{~cm}^{-3} \lesssim n<10^{6} \mathrm{~cm}^{-3}$, which shows that the CND is tidally unstable. The derived chemical composition is compared with a chemical model calculated using the UCL_CHEM code that includes gas and grain reactions, and the effects of shock waves. Models are run for varying shock velocities, cosmic-ray ionization rates, and number densities. The resulting chemical composition is fitted best to an extremely high value of cosmic-ray ionization rate $\zeta \sim 10^{-14} \mathrm{~s}^{-1}, 3$ orders of magnitude higher than the value in regular Galactic molecular clouds, if the pre-shock density is $n=10^{5} \mathrm{~cm}^{-3}$.
\end{abstract}

\section{Introduction}

The central molecular zone (CMZ) in the central few hundreds parsecs in the Galactic center hosts a large amount of molecular gas $\left(\mathrm{M} \gtrsim 2 \times 10^{7} \mathrm{M}_{\odot}\right.$; Oka et al. 1998). The interaction between the molecular mass and high-energy processes in the Galactic center region has been observed in many ways. Fermi/LAT captured high-energy $\gamma$-ray emission from the central regions of the Galaxy (Chernyakova et al. 2011), which suggests the presence of an enhanced cosmic-ray density. Observation of X-rays suggests that the supermassive black hole was active 100-300 years ago (Ponti et al. 2010 and Koyama et al. 1996). Chemical compositions of molecular clouds in the CMZ are likely to be affected by these high-energy activities. Sgr A* is surrounded by the circumnuclear disk (CND), a ring of molecular/atomic gas at a distance of 2-7 pc from the location of the supermassive black hole, whose chemistry can be most affected by the activity of Sgr A* Molecules with relatively strong emissions such as $\mathrm{CO}, \mathrm{HCN}, \mathrm{HCO}^{+}$have been previously observed both by single-dish and interferometers (e.g., Serabyn et al. 1986, Christopher et al. 2005). To study the overall chemical composition of the CND, a spectral line survey has been conducted that covers most of the frequency range between 80 and $500 \mathrm{GHz}$ that is observable from the ground (PI: Denise Riquelme). Here we outline how the observed data were analyzed in a framework of a chemical model to constrain the physical conditions in the CND. 


\section{Physical condition of the source}

Density estimates for the molecular clouds in the CND found in the literature vary greatly. Christopher et al. (2005) derive an average density of $n$, of $(3-4) \times 10^{7} \mathrm{~cm}^{-3}$ from their HCN $J=(1-0)$ emission map. Estimates by Montero-Castaño et al. (2009) also show densities of $n>10^{7} \mathrm{~cm}^{-3}$. On the other hand, a much lower density of $n \sim 10^{4}-10^{5}$ $\mathrm{cm}^{-3}$ was derived from multi- $J$ CO line observation by Requena-Torres et al. (2012). Relatively high temperatures of $T>200 \mathrm{~K}$ have been reported by Requena-Torres et al. (2012) and Bradford et al. (2005).

In the CND, sources of ionization may be cosmic-rays, X-rays, and UV-photons. Ionized atoms or molecules can induce ion-neutral reactions, which in general are faster than neutral-neutral reactions. UV-photons, which either come from OB stars or internally generated by cosmic-ray or X-rays, can efficiently dissociate molecules.

The value of the cosmic-ray ionization rate in most parts of the Galaxy is thought to be $\zeta \sim 3 \times 10^{-17} \mathrm{~s}^{-1}$ in dense molecular clouds and about an order of magnitude higher for diffuse clouds (Indriolo \& McCall 2012). For the CMZ, there are several claims of an elevated cosmic-ray ionization rate. In the dense cloud of the Sgr B2 region, van der Tak et al. (2006) show that the cosmic-ray ionization rate is $\zeta \sim 4 \times 10^{-16} \mathrm{~s}^{-1}$. Goto et al. (2008) find that the value in more diffuse CMZ material is $\zeta>10^{-15} \mathrm{~s}^{-1}$. There are even higher claims, such as $\zeta \sim 5 \times 10^{-13} \mathrm{~s}^{-1}$ (Yusef-Zadeh et al. 2007).

$\mathrm{X}$-rays affect chemistry in a similar way as cosmic-rays by a primary or secondary ionization followed by internal generation of UV-photons, which dissociate molecules. The supermassive black hole in Sgr A* currently has an X-ray luminosity, $L_{X}$, of $\sim 2 \times 10^{33} \mathrm{erg}$ $\mathrm{s}^{-1}$ in the $2-10 \mathrm{keV}$ range with the energy dependence $n(E) \propto E^{\Gamma}$ having a photon index $\Gamma$ of 2.7 (Baganoff et al. 2003). We estimate a value for the X-ray ionization rate using the method described in Maloney et al. (1996). The density of the cloud is assumed to be $n=10^{5} \mathrm{~cm}^{-3}$, and the X-ray flux at $E_{X}<2 \mathrm{keV}$ is assumed to have an energy dependence as $\exp (-2 \mathrm{keV} / \mathrm{E})$ and the minimum energy is taken as $0.1 \mathrm{keV}$. The X-ray ionization rate is extremely high $\left(\zeta \sim 10^{-14} \mathrm{~s}^{-1}\right)$ for the very edge of the cloud, but rapidly falls below the value of the cosmic-ray ionization rate in regular dense clouds in the Galaxy, which means that presently X-rays cannot be the dominant driving force of the chemistry although, possibly, a higher activity in the past may have had a strong impact.

Star clusters within the central parsec from Sgr A* are emitting ionizing photons at a rate of $2 \times 10^{50} \mathrm{~s}^{-1}$ (Lacy et al. 1980), producing photon-dominated regions (PDRs). Although a significantly enhanced photon field is expected, UV-photons can be attenuated with column densities of $N \sim 10^{22} \mathrm{~cm}^{-2}$. Since UV-photons may be attenuated deep in the molecular cloud, the effect of UV-photons are treated separately in our model.

\section{Observation and analysis}

Data was taken with IRAM 30 meter telescope and the FLASH receiver on the APEX telescope (for details, Riquelme et al., in preparation). Overlaid on the interferometer data of HCN $J=(4-3)$ by Montero-Castaño et al. (2009) in color scale, Figure 1 shows the position the southwest lobe of the CND towards which our line survey data were taken indicated by solid circles for the largest and smallest beam size of IRAM $30 \mathrm{~m}$, and dotted circles for the APEX FLASH receiver. The beam temperature is converted to a main brightness temperature scale assuming source sizes of $0.3 \mathrm{pc}$ from the interferometer data of Montero-Castaño et al. (2009). Along the same line of sight, there are multiple velocity components (50 $\mathrm{km} \mathrm{s}^{-1}$ cloud, $20 \mathrm{kms}^{-1}$ cloud, and CND), and only a nega- 


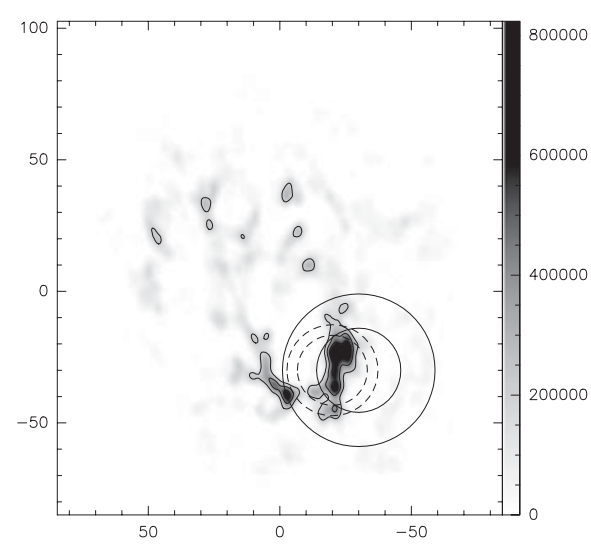

Figure 1: Grey scale shows the intensity of HCN $J=(4-3)$ emission from Montero-Castaño et al. (2009). Beam sizes of IRAM and APEX telescopes are shown in solid circles and dotted circles, respectively.

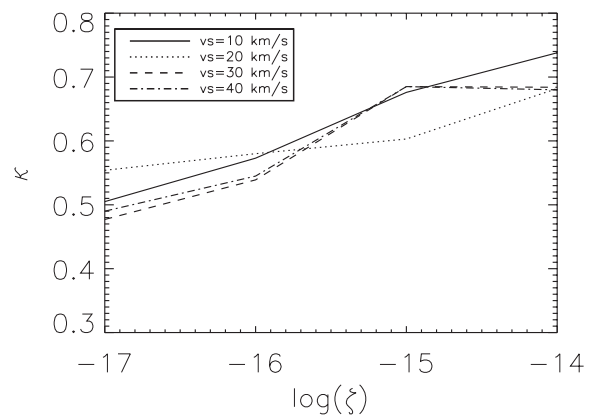

Figure 2: Values of confidence level with varying shock velocities are shown for the density $n=10^{5}$ $\mathrm{cm}^{-3}$.

tive velocity component, which certainly comes from the CND, is used for our analysis. Column densities for each species are derived employing a large velocity gradient (LVG) analysis based on the non-LTE radiative transfer code Radex (van der Tak et al. 2007). Column densities are obtained for $\mathrm{HCN} \mathrm{HCO}^{+}, \mathrm{HNC}, \mathrm{CS}, \mathrm{SO}, \mathrm{SiO}, \mathrm{CN}, \mathrm{H}_{2} \mathrm{CO}, \mathrm{HC}_{3} \mathrm{~N}$, $\mathrm{N}_{2} \mathrm{H}^{+}, \mathrm{H}_{3} \mathrm{O}^{+}$. The best-fit densities obtained by the LVG analysis for these species are $10^{5} \lesssim n<10^{6} \mathrm{~cm}^{-3}$.

\section{Chemical models}

We used time-dependent gas-grain chemical model UCL_CHEM Viti et al. (2004), which was extended to include the effect of shock waves Viti et al. (2011). The model calculations were run for different final densities $n=10^{4}, 10^{5}$, and $10^{6} \mathrm{~cm}^{-3}$, cosmic-ray ionization rates $\zeta=10^{-17}, 10^{-16}, 10^{-15}$, and $10^{-14} \mathrm{~s}^{-1}$, and shock velocities of 10,20 , 30 , and $40 \mathrm{~km} \mathrm{~s}^{-1}$. Different sets of elemental abundances were used, including the "lowmetal" abundances and solar abundances described in Wakelam \& Herbst (2008). The overall match with the observations is examined by a parameter $\kappa$ used in Garrod et al. $(2007) ; \kappa_{\mathrm{i}}=\operatorname{erfc}\left[\left|X_{i, \text { calc }}-X_{\mathrm{i}, \mathrm{obs}}\right| /(\sqrt{2} \sigma)\right]$. Figure 2 shows values of $\kappa$ in each model at its time of best agreement. The overall agreement with the observations is better with a high value of cosmic-ray ionization rate $\zeta=1 \times 10^{-14} \mathrm{~s}^{-1}$ for all the densities, but the $\zeta$-dependence is weaker when $n=10^{4} \mathrm{~cm}^{-3}$. The best agreement in chemistry is achieved when $n=10^{4} \mathrm{~cm}^{-3}$, which is lower than the density derived by the LVG analysis.

Figure 3 shows fractional abundances of selected species as a function of time from the time of shock passage. Although for most species, the values are in good agreement with the observation at the time of best agreement at $t=10^{4} \mathrm{yr}$, CN and CS are underabundant by more than an order of magnitude. The fractional abundance of CN can be enhanced in PDRs. There is also an observational claim that sulfur elemental abundances are higher in PDRs because of less depletion of sulfur on dust grains (Goicoechea et al. 2006). 

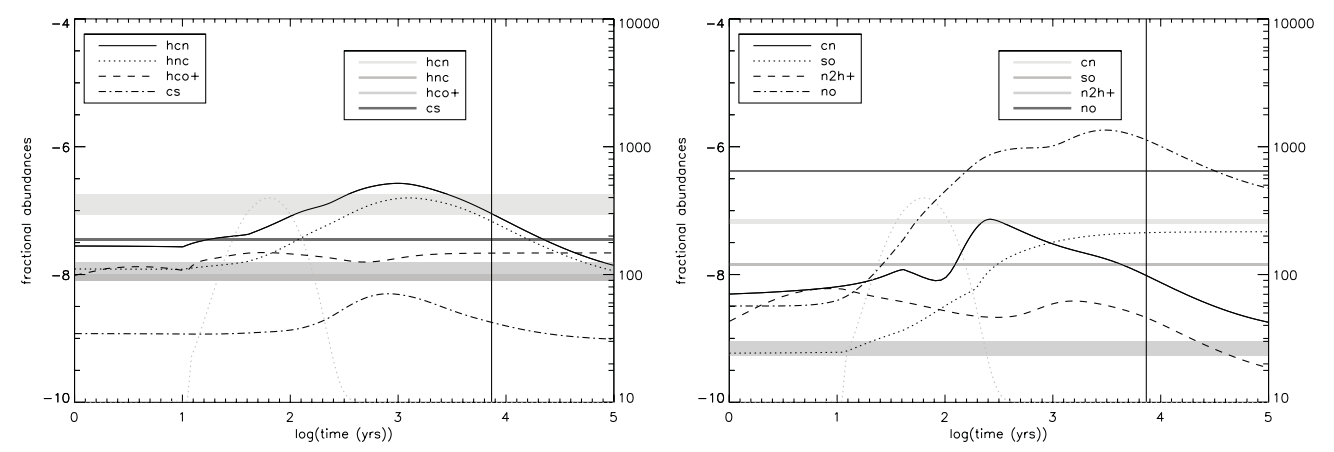

Figure 3: Calculated fractional abundances averaged over time from the time of shock passage and observed values are shown for $n=10^{5} \mathrm{~cm}^{-3}, \zeta=10^{-14} \mathrm{~s}^{-1}$, and $v_{s}=$ $20 \mathrm{~km} \mathrm{~s}^{-1}$.

\section{Discussion and summary}

Our results show that, at the time of best agreement $\left(10^{4} \mathrm{yr}\right)$, the observed chemical composition fits chemical models best when the cosmic-ray ionization rate at the circumnuclear disk is $\zeta=1 \times 10^{-14} \mathrm{~s}^{-1}$, about three orders of magnitude higher than the regular galactic molecular clouds. Although previous work has suggested the value similar to our results, the latter should be taken with caution. Our parsec-scale size beams cover multiple molecular cloud components that are resolved in interferometer maps, and that have varying densities, while our chemical models are run only for a fixed density in each model. The time of best agreement we derived happens to correspond to the time of cosmic-ray turn on suggested by the model of Chernyakova et al. (2011).

\section{References}

Baganoff, F. K., Maeda, Y., Morris, M., et al. 2003, ApJ 591, 891

Bradford, C. M., Stacey, G. J., Nikola, T., et al. 2005, ApJ 623, 866

Chernyakova, M., Malyshev, D., Aharonian, F. A., Crocker, R. M., \& Jones, D. I. 2011, ApJ 726,60

Christopher, M. H., Scoville, N. Z., Stolovy, S. R., \& Yun, M. S. 2005, ApJ 622, 346

Garrod, R. T., Wakelam, V., \& Herbst, E. 2007, A\& A 467, 1103

Goicoechea, J. R., Pety, J., Gerin, M., et al. 2006, A\&A 456, 565

Goto, M., Usuda, T., Nagata, T., et al. 2008, ApJ 688, 306

Indriolo, N. \& McCall, B. J. 2012, ApJ 745, 91

Koyama, K., Maeda, Y., Sonobe, T., et al. 1996, PASJ 48, 249

Maloney, P. R., Hollenbach, D. J., \& Tielens, A. G. G.. M. 1996, ApJ 466, 561

Montero-Castaño, M., Herrnstein, R. M., \& Ho, P. T. P. 2009, ApJ 695, 1477

Lacy, J. H., Townes, C. H., Geballe, T. R., \& Hollenbach, D. J. 1980, ApJ 241, 132

Oka, T., Hasegawa, T., Hayashi, M., Handa, T., \& Sakamoto, S. 1998, ApJ 493, 730

Ponti, G., Terrier, R., Goldwurm, A., Belanger, G., \& Trap, G. 2010, ApJ 714, 732

Requena-Torres, M. A., Güsten, R., Weiß, A., et al. 2012, A\&\&A 542, L21

Serabyn, E., Guesten, R., Walmsley, J. E., Wink, J. E., \& Zylka, R. 1986, A\& A 169, 85

Tielens, A. G. G.. M. 2005, The Physics and Chemistry of the Interstellar Medium A. G. G. M. Tielens, ISBN 0521826349, Cambridge, UK: Cambridge University Press, 2005.,

van der Tak, F. F. S., Belloche, A., Schilke, P., et al. 2006, A\&SA 454, L99

van der Tak, F. F. S., Black, J. H., Schöier, F. L., Jansen, D. J., \& van Dishoeck, E. F. 2007, $A \mathscr{E} A 468,627$ 
Viti, S., Collings, M. P., Dever, J. W., McCoustra, M. R. S., \& Williams, D. A. 2004, MNRAS 354,1141

Viti, S., Jimenez-Serra, I., Yates, J. A., et al. 2011, ApJ Lett. 740, L3

Wakelam, V. \& Herbst, E. 2008, ApJ 680, 371

Yusef-Zadeh, F., Muno, M., Wardle, M., \& Lis, D. C. 2007, ApJ 656, 847 